\title{
CONVENIO ENTRE LA FEDERACIÓN DE NIGERIA Y LA PROVINCIA ESPANOLA DEL GOLFO DE GUINEA
}

\author{
Javier Martínez Alcázar \\ Embajada de España en Lagos. Nigeria.
}

\section{I.-ANTECEDENTES A MODO DE PREÁMBULO.}

España había prestado muy poca atención a los territorios españoles de Fernando Poo y Annobón, en el Golfo de Biafra, desde que se adquirieron por el Tratado de San Ildefonso firmado con Portugal en $1777^{\prime}$, a cambio de un nuevo trazado de fronteras en Brasil. Prácticamente estos territorios permanecieron en el mismo estado en que se encontraron hasta 1783 en que el conde de Argelejos partió desde Montevideo hacia Fernando Poo, en una expedición que fracasó totalmente. Hasta esta fecha, pues, no hubo en la isla de Fernando Poo presencia alguna española aunque sí británica, ya que la marina de este país había logrado fijar una base en Fernando Poo visitando la isla en 1783 el Comodoro inglés Busler. Dichas expediciones inglesas continuaron durante todo el siglo XIX, en 1819 la de Robetson, en 1821 la de Kelly y en 1827 la de Owen que funda Port Clarence, que España rebautizará en 1845 como Santa Isabel. Todas estas expediciones inglesas tenían como objetivo recabar información sobre las posibilidades de explotación de la isla y posterior intento de hacerse con ellas.

El Gobierno inglés, ya bien asentado en Fernando Poo, propuso al Gobierno de Madrid la adquisición de dichos territorios a cambio de una isla británica en el Caribe ${ }^{2}$. Desde Madrid se dio largas al asunto, sin que tampoco se tomase ninguna decisión para asentar su soberanía sobre los territo- 
rios que le correspondían en Guinea Ecuatorial, hasta que en 1832 se nego a ceder la soberanía de la isla.

El primer intento serio de acercamiento por parte de España hacia los territorios de Guinea Ecuatorial partió de la Cortes Constituyentes reunidas en tiempo del general Prim, que en 1869 hicieron una interpelación al Gobierno sobre la situación de abandono en que se encontraban dichos territorios. De este asunto tuvo conocimiento un hombre especial e inquieto que estaba destinado como Cónsul General de España en Freetown, Sierra Leona, Don José Manuel de Echeverri, quien en 1870 tuvo la iniciativa de dirigir una memoria al Gobierno de Madrid titulada "Memoria que contiene algunos datos con cuyo auxilio tal vez se facilite la resolución del tema propuesto en las Cortes Constituyentes referente a si es o no conveniente para España la posesión, bajo su dominio, de la Ysla de Fernando Poo" ${ }^{3}$.

Echeverri estaba convencido que la explotación de la isla de Fernando Poo podía ser rentable para España y que en muy pocos años estos territorios podrían ser muy beneficiosos para la economía española, al igual que los británicos habían conseguido de sus territorios en Sierra Leona. "Daré, afirmó, mi pobre parecer sobre este particular, toda vez que tanto los españoles como el Gobierno que hoy rige á España desean se verifique cuanto antes la emancipación de la esclavitud odiosa que existe en la Ysla de Cuba, dese principio por verificarlo con el insignificante número de tres mil y con ellos cópiese en todas sus partes las resoluciones y la egecución del Gobierno inglés que ecsistía el año $1792 " 4$.

En su tesis, Echeverri defendía la idea de reclutar obreros libres para con ellos colonizar los territorios ecuatoriales, como ya habla sido aplicado por Gran Bretaña en el Caribe y Sierra Leona. Echevarri propone, en definitiva, dar trabajo a la población de color que al dejar de ser esclava se encuentra en gran medida desorientada y es gente bien experimentada para todo tipo de trabajos. Sin embargo, el Gobierno de Madrid no tomó ninguna disposición seria con respecto a Fernando Poo hasta el envío de la expedición de Don Manuel Iradier que procedió a la efectiva ocupación de aquellos territorios entre 1875 y $1884^{\mathrm{s}}$.

El Gobierno de la República, por boca de su Presidente Alcalá Zamora, había declarado su firme propósito de hacer rentables los territorios de Guinea. Para ello se dictaron una serie de circulares ministeriales en las que se reseñaban una serie de normas para la mejora de las plantaciones y las bases para la contratación de mano de obra de los territorios cercanos. Así, por el Estatuto de 1931 la República plasmó su código de intenciones, tan sencillo como ambicioso: democratización, intentos de autofinanciación y selección de funcionarios eficaces. Pero el principal problema, el de la falta 
de mano de obra, continuaba sin resolver. Por ello, el Gobierno firmó en 1934 un tratado con las autoridades francesas de Camerones (Camerún).

En síntesis, en el Tratado 6 se fijaban las condiciones para la recluta de súbditos de Camerones solicitados por las empresas españolas en las posesiones de Guinea bajo las siguientes cláusulas:

1.- Serían rechazados todos los indígenas que hubiesen entrado sin permiso en los territorios españoles.

2.-Todos aquellos que ya se encontrasen en el territorio y no pudieran renovar sus contratos de trabajo serfan repatriados a Camerones por cuenta de las Autoridades españolas.

3.-El Gobierno español fijaría anualmente el número de braceros necesario para las plantaciones y explotaciones españolas, que no podría superar la cifra de cuatro mil.

4.-El Gobierno francés confeccionaría una lista con aquellos braceros que estuviesen autorizados a emigrar, a los que se facilitaría un salvoconducto y una ficha médica con su estado sanitario.

5.-Los gastos del transporte de cada trabajador voluntario, serían costeados por el Gobierno francés hasta Douala, puerto marítimo, o hasta la frontera terrestre de Amban. El viaje desde estos puntos hasta Guinea sería costeado por las Autoridades españolas.

6.-Antes del embarque se formalizaría un contrato con cada trabajador y el representante español acreditado en Douala en el que, entre otras condiciones, se fijaría un salario de veinte pesetas mensuales. Los contratos serían por dos afios con la posibilidad de renovación hasta un máximo de seis.

7.-Al término del contrato los braceros serían repatriados a sus lugares de origen por cuenta de los patronos, y con la garantía del Gobierno español.

8.-Por último, se autorizaba al representante del Gobierno francés en Santa Isabel para que hiciese el seguimiento del cumplimiento de los contratos y de que las condiciones de trabajo fuesen correctas, recordando la prohibición absoluta de beber alcohol.'

Pero ya sabemos que el 18 de Julio de 1936 las cosas cambiaron radicalmente para España, y Guinea Ecuatorial iba, una vez más, a estar olvidada por todos. Con todo, la cifra más aproximada de cameruneses que se habría trasladado a trabajar a las plantaciones de Guinea no superó, en ningún momento, los 2.000 y casi todos ellos permanecieron en la región continental donde ya vivian algunos de sus familiares que habrían emigrado con anterioridad. 
Tras el paréntesis de la guerra civil española y las primeras indecisiones de Franco que tardaron más de quince años en plasmarse en algo concreto, lo cierto es que será bajo este régimen cuando se decida la verdadera colonización de los territorios de Guinea Ecuatorial.

En 1959 se inició la fase de provincialización de dichos territorios, y con ello el desarrollo socioeconómico alcanzó importantes cotas hasta el punto de que los propios ingresos coloniales financiaban el presupuesto de Guinea Ecuatorial sin que el mismo costase una sola peseta al presupuesto nacional. Resumiendo, se puede afirmar que la etapa que va de 1949 a 1959 se caracterizó por ser la más floreciente de la colonización española en los territorios que fueron asignados a España por el Tratado de Paris de $1900^{\circ}$.

\section{II.-CONSIDERACIONES ACERCA DE LA NECESIDAD DE FIR- MAR ACUERDOS LABORALES PARA LA CONTRATACION DE MANO DE OBRA PARA LAS PLANTACIONES DE FERNANDO POO.}

Hemos visto el el escaso interés de los diferentes gobiernos de Madrid por sus posesiones de Guinea Ecuatoriala lo que había que añadir el problema de la despoblación en que se encontraban dichas territorios, lo que dificultaba enormemente su explotación. En 1905 se firmó el primero de una serie de acuerdos comerciales tendentes a conseguir mano de obra en la cercana costa africana para emplearla en las incipientes plantaciones de Fernando Poo. Como consecuencia de este tratado con el Gobierno de Liberia ${ }^{9}$ llegaron a principios de siglo a Fernando Poo unos 300 liberianos que se adaptaron perfectamente a los condiciones de vida en la isla dando así origen a una población oriunda de Liberia que aún persiste en la actualidad.

Un 1951 el Gobierno español inició conversaciones con el británico, que en esa fecha era la potencia colonizadora de Nigeria, para la creación de la Agencia Hispano-Inglesa de empleo, cuya finalidad sería facilitar la contratación de braceros nigerianos, que voluntariamente, quisiesen trasladarse a trabajar en las explotaciones agrícolas españolas de Guinea Ecuatorial. Fruto de estas conversaciones fue el Primer Convenio Laboral firmado por los Gobiernos de Inglaterra en Nigeria y las Provincias Españolas del Golfo de Guinea del 6 de Septiembre de $1957^{10}$.

En este primer Convenio se fijaron las bases para la regulación de la contratación de los braceros nigerianos para las explotaciones agrícolas de Fernando Poo. En los convenios sucesivos firmados con el Gobierno español de 1958 y 1963, y los firmados entre el Gobierno de Nigeria y la República de Guinea Ecuatorial, las condiciones de dicha contratación de los braceros nigerianos variaron poco. 
La recluta de los braceros nigerianos para el trabajo en las explotaciones agrícolas de las provincias españolas de Río Muni y Fernando Poo, se realizaba en el Este de Nigeria , sobre todo de la región de Calabar, por lo que estos trabajadores eran conocidos como calabares y no como nigerianos ".

En los tratados firmados hasta 1957 y en los posteriores se resaltó siempre el carácter voluntario de estas contrataciones, lo que en gran medida era cierto, ya que la situación económica de Nigeria en esta época no podía absorber toda la mano de obra que había excedente. La contratación se hacia por dos años y al terminar el contrato tenían opción para quedarse un año y medio más siempre que la empresa contratante estuviese satisfecha con su trabajo. La forma de pago era algo complicada ya que cada bracero percibía 700 pesetas mensuales, de las cuales 445 se les entregaban en mano y 225 en moneda nigeriana a su retorno. Además se les proveía de raciones de arroz, malanga, pescado seco, sal y aceite por un valor aproximado de 60 pesetas mensuales. Se les proporcionaba una pequeña casa de cemento y de una pequeña parcela de tierra para cultivar alguna cosa. A este sueldo mensual había que añadir 3.600 pesetas que la empresa les paga por la recluta.

Las empresas españolas de Guinea Ecuatorial estaban muy satisfechas con el rendimiento de estos obreros y prueba de ello es en las plantaciones de Fernando Poo se obtenían mejores resultados que en las de Rio Muni donde el porcentaje de estos obreros era mucho menor.

\section{III.-PROBLEMAS POLITICOS DE LA CONTRATACIÓN.}

La prensa nigeriana siempre mostró una actitud muy contraria a la firma de los convenios laborales entre Nigeria y España, acusando a las autoridades españolas de malos tratos a los braceros y de usos y maneras coloniales. Pese a ello, y desde las propias instancias del Gobierno nigeriano, la postura era favorable a estos contratos. Una información de primera mano la proporciona el propio Cónsul de Nigeria en Santa Isabel quien en 1962 afirmaba que "las condiciones de trabajo en Fernando Poo no son tan negativas como las descritas por la prensa de mi país, pudiéndose incluso hablar de condiciones atractivas para el trabajador" ${ }^{12}$. Podríamos afirmar que una de las razones que llevaría a la prensa nigeriana a orquestar esta campaña contra España, estaría en el hecho de entorpecer y enturbiar la política progresista del Primer Ministro de la Federación Nigeriana Sr. Abubakar ${ }^{13}$.

El 30 de Mayo de 1962 y durante el Congreso del Action Group en la ciudad de Jos, un grupo de estudiantes extremistas de la Universidad de dicha ciudad, acusaron al Gobierno nigeriano de confabulación con el Gobierno 
español, por su acción en Fernando Poó. La postura del Gobierno de Nigeria se vio debilitada por los acontecimientos de aquellas fechas ya que en la Conferencia de Jefes de Estado en Lagos que, aunque no alcanzó los objetivos perseguidos se actuó como revulsivo para los sentimientos nacionalistas y los deseos de autodeterminación para todos los países africanos y la posición de Rusia y Estados Unidos que, manifestándose favorables a estos principios de autodeterminación, reciben de ellos el apoyo moral y eventualmente el material de guerra necesario para sus intereses.

También en 1962, el "Nigerian Youth Congress", como conclusión de una de sus sesiones de trabajo, criticó duramente la presencia de España en Fernando Poó, aludiendo al carácter colonial de la presencia española allí, reinvidicando la isla de Fernando Poo como una porción de Nigeria. Esta postura también era apoyada por algunos sectores oficiales que abogaban por el favorecimiento de este éxodo de obreros, con la idea de que una vez que los nigerianos en la isla sean la aplastante mayoría, sería más fácil conseguir lo que por razones geopolíticas ya les pertenece. Sin embargo, y en opinión del Embajador de España en Lagos ${ }^{14}$, no consideraba que la masa de nigerianos que trabajan en la isla, desorganizada e ignorante, pudiese significar un peligro para España, desde dentro de la isla. El problema podría venir que si los nigerianos siguen manteniendo esta clara superioridad, ello podría ser utilizado para plantear la cuestión desde fuera de ella, sea por grupos de Estados Africanos o por las Naciones Unidas. Por ello, nuestro Embajador sugiere que, teniendo en cuenta el rumbo que están tomando las cosas en Africa y en vista del interés que hay en crear problemas donde no los hay, quizá fuera aconsejable desposeer a Nigeria de esta mayoría de la que tanto blasona y diversificar la recluta de obreros hacía otros países africanos. Finalmente y en opinión asimismo de nuestro Embajador, la sugerencia más práctica sería potenciar una emigración española ordenada y cualificada hacia aquellas provincias ecuatoriales. El clima y las condiciones sanitarias han mejorado muchísimo y ningún blanco debe temerlas. La metrópoli, afirma nuestro Embajador, podría así tener un pié firme en ultramar y dado que la población nativa es tan exigua quizá pudiera alterarse así el equilibrio poblacional a favor de los españoles. No debe olvidarse que en el despertar de Africa, nuestra isla, estratégicamente situada en el Golfo de Biafra, rozando Camerún y Nigeria, podría estar llamada a grandes posibilidades y mayores destinos ${ }^{15}$.

El 17 de Agosto de 1962 en el Parlamento Federal de Nigeria, al debatirse el problema del contrabando, un diputado aludió a la isla de Fernando Pob como una de las bases para introducir fraudulentamente mercancías en el país. Insistiendo en dicha afirmación el diputado Sr. F.C. Ogbalu, sugirió 
que Nigeria estableciera negociaciones con el "poder colonialista" para entrar en posesión de la isla o que se procediera simplemente a su conquista como en el caso de Goa ocupada por las fuerzas de la India. Dicha declaración, aunque tiene su importancia por haber sido hecha en el Parlamento, no tuvo más trascendencia al no haber encontrado eco ni entre los demás diputados ni en el Gobierno.

Lo cierto es que, por unas u otras razones, el tema de España y los braceros encontraba cada vez más eco entre la población nigeriana, extremo que era bien aprovechado por los extremistas. Por todo ello, hay que resaltar la declaración que a este respecto hizo ante un grupo de estudiantes de la Universidad de Ibadán, el Primer Ministro Abubakar, al prevenirles contra la siembra de ideas demagógicas, que abusando de su juventud, siembran en ellos apetencias políticas sobre Fernando Pob. Por otra parte, afirmó, que no era cierto que Nigeria tuviese ningún plan para la invasión de Fernando Poó, negando que pudiesen existir intereses encontrados de Nigeria, Camerún y Gabón, al respecto ${ }^{16}$.

Todo ello, puede contribuir a explicar la audacia e irresponsabilidad con que actúan estos extremistas que desencadenaron la violenta campaña por la cuestión de Fernando Poó, dirigiendo sus amenazas tanto contra el Gobierno Federal de Nigeria, como contra España.

\section{IV.-DATOS PARA NEGOCIAR LA REVISION DEL CONVENIO LABORAL DE BRACEROS.}

Ante la inminencia de los primeros contactos entre Nigeria y España para negociar un nuevo convenio laboral de contratación de braceros, parece oportuno considerar las opiniones de nuestro Ministro Encargado de Negocios en Lagos quien afirmaba que en dicha negociación había, en cierta manera, intereses contrapuestos, ya que el interés del Estado español, en el momento actual, debía ser el de suavizar las asperezas de esta engorrosa cuestión que podría traer repercusiones políticas negativas para España. Por ello, el primer paso por parte de España debía ser el de mejorar sustancialmente el Convenio de $1958^{17}$.

Entre toda la documentación que he consultado, previa a la negociación del Convenio de 1963, se deduce que las tres partes implicadas, es decir, el Gobierno de Nigeria, el Estado espaniol y los empresarios españoles de Fernando Poó acudieron a esta negociación animados con el mejor espíritu conciliatorio y deseosos de llegar pronto a un acuerdo.

Para los propietarios, lógicamente atentos al lucro y a reducir lo más posible sus costes, las condiciones económicas no se debían mejorar dema- 
siado, a no ser que, como contrapartida, se alargase el plazo de estancia de los braceros en la isla hasta tres años ${ }^{18}$ para de esta forma, resarcirse de los gastos generales de contratación de cada bracero que alcanzan, como sabemos, más de 3.600 pesetas. También y, como segunda contrapartida, estarían dispuestos a mejorar las ya adecuadas condiciones de habitabilidad en las plantaciones. Para los propietarios es, asimismo, importante que cada bracero vaya acompañado únicamente por una mujer extremo que apoyarían, incluso, los propios nigerianos que saben que si llevan varias mujeres con ellos acaban dedicándose a la prostitución. En cuanto al tema de las mejoras económicas los madereros de Río Muni manifestaron que no estaban en condiciones de mejorarlas ya que sus explotaciones no rendían lo suficiente, al contrario que las de Fernando Poó en las que se obtenían cuantiosos beneficios.

España, por su parte, debía exhibir alguna mejora, aunque sólo fuese de tipo propagandístico, con el fin de ayudar al Gobierno nigeriano en los ataques que recibe de la prensa de su país. Convendría, por ejemplo, mejorar todo el sistema de multas a los braceros y los reintegros que por estos conceptos deben hacer al patrono por faltas. Lo más práctico sería estudiar las condiciones para repatriar a los indeseables que, dada la cercanía de Nigeria, se podría hacer a muy bajo coste.

\section{V.-NEGOCIACIONES DEL CONVENIO DE BRACEROS DE 1963.}

La primera gestión para la negociación del nuevo convenio de braceros se hizo a iniciativa nigeriana, quien por Nota Verbal número 822 de 12 de Agosto de 1961, instaba al Gobierno español a enviar a Lagos a una delegación cualificada para examinar y firmar el Convenio de trabajo sobre los braceros nigerianos en Fernando Poo ${ }^{19}$. Por las mismas fecha, el ministro de Trabajo de la Federación de Nigeria, Sr. Johnson realizó una visita de trabajo a Fernando Poó para inspeccionar algunas haciendas e instituciones nigerianas de ayuda al trabajador, manteniendo contactos con las autoridades españolas y nigerianas en la isla. A su regreso a Lagos el Ministro hizo unas declaraciones a la prensa bastante favorables respecto a las condiciones de trabajo de los braceros. La actual y urgente demanda del Gobierno nigeriano respondía a la necesidad de llevar a cabo actos de soberanía con que justificar su recién ganada independencia y no precisamente porque el problema revista características especiales ni de importancia ni de urgencia económica ${ }^{20}$.

Posteriormente por otra Nota Verbal número 2.314 se insiste en que dichas negociaciones deben realizarse en Lagos, como conveniente para los 
intereses de Nigeria, para acallar el ambiente de las últimas campañas de malos tratos a los braceros que culpaban al Gobierno de la Federación de ser poco firmes. Por tercera vez, y sin que hasta esa fecha haya habido ninguna respuesta a las demandas nigerianas, Nigeria insiste continuamente y por todos los medios a su alcance en su deseo de que una delegación española venga a Lagos a negociar el convenio de braceros.

No se comprende demasiado el interés de esta negociación como no sea que se deba a que el Convenio de 1958 fue firmado por la entonces autoridad británica y a los problemas de exceso de mano de obra que Nigeria tiene en el Este del país, de donde procede la mayoría de los braceros. Aún con todo, la insistencia en firmar un nuevo convenio era, ya, un verdadero clamor que estaba empezando a molestar a las autoridades nigerianas ante el silencio del Gobierno español.

A estas tres peticiones el Ministerio de Asuntos Exteriores español contestó por telegrama cifrado número 12 de 17 de Noviembre de $1962^{21}$, aceptando las condiciones para la negociación del nuevo convenio de braceros y prometiendo que una delegación española se desplazaría a Lagos en Enero de 1963. El Ministro de Trabajo nigeriano, en conversación telefónica con nuestro Representante Diplomático en Lagos, aceptó las condiciones impuestas por España sugiriendo que habría que cambiar algunas cláusulas, coincidiendo con España que, tras una Nigeria independiente, era bueno para ambas partes la conclusión de un nuevo tratado que obligase a dos estados independientes y soberanos.

Habĺa que tener en cuenta que el planteamiento de esta negociación era muy anterior a la actual crisis política que atravesaba Nigeria. El Ministro de Trabajo, vivo exponente de los nacionalismos y ambiciones de la época, mostraba un espíritu más flexible y comprensivo que España, que se encontraba en una situación de privilegio para esta negociación ante el estancamiento del inoperante Plan de Desarrollo que Nigeria estaba intentando llevar a cabo. Es de destacar, incluso, el hecho de que la agresiva prensa nigeriana guardaba el más absoluto silencio ante estas negociaciones lo que habría que interpretar como si ésta hubiese recibido un "mot d'ordre" para alejarse de esta cuestión ante la clara visión del Ministro Johnson de la conveniencia de evitar que la misma fuese utilizada por los extremistas y demagogos que dificultasen una conciliación que, en definitiva, favorecía más a Nigeria que a España dado que la isla de Fernando Poó estaba habitada por aquella época por más de 30.000 nigerianos.

Las negociaciones comenzaron, finalmente, en Marzo de 1963 y por indicación del propio Ministro Federal de Trabajo éstas se llevaron a cabo por un grupo reducido de tres personas por cada parte. Esta idea fue muy favo- 
rable a España al eliminar un buen número de personas con lo que se simplificaban las discusiones y podria transcurrir todo en mayor orden.

La Delegación nigeriana demostró un gran interés por la rápida firma del Convenio ya que el nuevo convenio, insisto, derogaría el firmado con anterioridad por la Autoridad Británica, garantizaría la permanencia en la isla de los más de 30.000 nigerianos que allí residían y, en definitiva, supondría una entrada de divisas en Nigeria.

Pese a todo este buen ambiente inicial, las negociaciones fueron muy laboriosas, por el tesón con que la Delegación nigeriana quería proteger los intereses de sus braceros aumentando las facultades de su Cónsul y de los Agregados laborales, planteando reclamaciones que eran inaceptables para España. De esta forma, las negociaciones tomaron un mal cariz por las reclamaciones que el Cónsul de Nigeria en Santa Isabel planteaba en las mismas ${ }^{22}$. El Ministro Sr. Johnson zanjó la cuestión desautorizando a su propio Cónsul.

Por parte de España se insistió en el hecho de que la idea de que el envio de trabajadores nigerianos a Fernando Poo fuese una reminiscencia de épocas pasadas, creadora de abusos y tensiones era, falso. Se argumentó sobre las importantes corrientes laborales existentes en la actualidad en Europa que se consideran como muy beneficiosas para la aproximación entre los diferentes pueblos y una mejor distribución de la riqueza, en la seguridad que este nuevo convenio debía repercutir en beneficio de ambos países.

Terminado el trabajo en Comisión, y en una sesión del Pleno, presidida por el Ministro y el Embajador de España, se abordaron los pequeños puntos en discordancia. El texto se aprobó, finalmente, con resultados más favorables para España que el Convenio de 1958, estando limpio de expresiones atentatorias a la Soberanía espanola sobre aquellos territorios y cercenando las pretensiones del Cónsul y de los Agentes laborales nigerianos. No se tocó la cuestión salarial para respetar un Acuerdo que se hizo en 1961 de que se mantendría su vigencia durante tres años. Por ello, la Delegación nigeriana se reservó el derecho a plantear la cuestión el año próximo.

El lunes primero de Abril de 1963 se terminaron los trabajos regresando los dos miembros de la Delegación española, Sres. Fernando Morán, Primer Secretario de Embajada, y Esteban Hanza, Delegado de Trabajo en Santa Isabel, a Fernando Poo.

Los textos aprobados se remitieron por valija diplomática conducida a Madrid para que fueran convenientemente estudiados por el Ministerio de Exteriores y, una vez dada su conformidad, enviar la correspondiente Plenipotencia al Embajador de Espania en Lagos para su firma. Finalmente, el Convenio se firmó y selló el 18 de Mayo de 1963 en el despacho del 
Ministro Johnson, quien pronunció unas palabras de agradecimiento a la parte española con el deseo de que el nuevo Convenio se llevase al mejor cumplimiento en todas sus cláusulas, lo que redundaría en beneficio de ambas partes.

\section{VI.-DATOS SOBRE LOS PROBLEMAS QUE AFECTAN A LOS BRACEROS NIGERIANOS EN FERNANDO POO.}

Como se ha venido afirmando a lo largo de esta exposición, no parece que el trato que los braceros nigerianos recibían en las plantaciones españolas de Fernando Poo fuese especialmente duro, aunque su trabajo en sí, sí lo era. Prueba de ello es que a lo largo de muchos años no se produjeron incidentes graves.

Nigeria mantenía en Santa Isabel un Cónsul y una serie de Agregados laborales que estaban encargados de la supervisión de todo lo relativo a los braceros nigerianos, sin tener sobre ellos ninguna autoridad ya que necesariamente tenían primero que informar al Gobierno General de la isla. Desde Agosto de 1963, es decir, en las fechas en que se estaba preparando el nuevo convenio, ocupaba el cargo de Cónsul el Sr. Christopher Chike Chukwa, que había sustituido al Sr. Ikemefuna, que tantos espinosos problemas había creado a las autoridades españolas de la isla.

Entre los incidentes ocurridos cabe señalar como grave la equivocada detención del Cónsul nigeriano en Santa Isabel, Sr. Chuwua por las autoridades de la isla. Este, independientemente de que su actuación en medio de unos grupos subversivos nigerianos no estaba demasiado justificada, gozaba de inmunidad diplomática y por ello, en ningún caso, se le podía detener. Como consecuencia de ello, el Ministerio de Asuntos Exteriores nigeriano, elevó una enérgica protesta ante las autoridades españolas, y entre las expresiones reflejadas en dicha Nota Verbal se emplearon algunas desafortunadas como "man-handling", "gun-point", "heavily armed spanish soldiers" y en modo alguno se podía admitir "cases of inhuman treatment of nigerians by Spanish officials". Ante esta nota, el Embajador de España en Lagos solicitó y no obtuvo permiso de Madrid para hacer patente la protesta de España ante los insultos vertidos por las autoridades nigerianas. Por ello, el Ministerio de Asuntos Exteriores español quizá, empleando las técnicas dilatorias que por aquella época se utilizaban en España consideró más oportuno dar largas al asunto y dejar que el mismo se enfriase solo.

El 15 de Marzo de 1963 se produjo otro incidente, esta vez con uno de los Agregados laborales, que acusó a España de haber sido apaleado por la policía, hechos que no pudieron ser probados. 
Otra Nota Verbal del Ministerio nigeriano de Asuntos Exteriores pedía explicaciones sobre la muerte de un bracero nigeriano ocurrida en una de las plantaciones españolas. Se contestó que dicho bracero había mostrado una actitud agresiva y culpable que saliéndose del ámbito laboral llamó a la polić́a de la isla que se encontró con un pequeño motín, y en la refriega resultó muerto dicho bracero. Instruido el correspondiente sumario por la autoridad civil se concluyó que el policía había actuado dentro de sus funciones y que por lo tanto estaba exento de culpabilidad.

Concluyo afirmando que, tras muchas indagaciones efectuadas personalmente con ex-braceros nigerianos con los que he podido conversar en Lagos, la impresión es que todos recuerdan aquella época como una de las mejores de su vida, entre otras cosas, me dicen, porque el trabajo no era excesivamente duro y el salario muy bueno, lo que les permitía ayudar a la familia que residía en Nigeria.

\section{NOTAS}

1 Los Tratados de San Ildefonso y de El Pardo respectivamente del 1 de Octubre de 1777 y de 24 de Marzo de 1778, firmados entre Portugal y Espania, lo lo que Portugal cedía a Espania las islas de Fernando Poo y Annobón a cambio de la Colonia espafiola de Sagramento

2 Archivo del Ministerio de Asuntos Exteriores. Legajo 4/94

3 Archivo del Ministerio de Asuntos Exteriores. Legajo 2.066. Memoria Echeverri.

4 Archivo del Ministerio de Asuntos Exteriores. Legajo 2.066. Memoria Echeverri.

5 El vasco Manuel Iradier realizó dos viajes a Guinea Ecuatorial. El primero entre 1875 y 1876 y el segundo en 1884. Iradier exploró toda la región del estuario del Muni y del cabo San Juan: explorando todos los tios, bosques, islas y poblados. Los trabajos de este insigne hombre hicieron posible que Espana pudiese mantener su presencia en Guinea, cuando los franceses se estaban apodarando con todo.

6 Tratado de Camerones firmado entre el Gobernador General de los Territorios espanioles del Golfo de Guinea y el Comisario de la República francesa en Camerones, debidamente autorizados por sus gobiernos respectivos, de 8 de Enero de 1934.

7 La República prestó mucha atención al tema de la contratación de mano de obra de fuera de Guinea, y prueba de ello es la abundante legislación que, a este respecto, produjo: Aviso de 23 de Junio de 1934. Decreto de 27 de Septiembre de 1934. Disposición de 22 de Noviembre de 1934. Instrucción de 25 de Noviembre de 1934. Circular de 12 de Diciembre de 1934. Decreto de 31 de Diciembre de 1934. Oficio de 12 de Enero de 1935. Decreto de 12 de Febrero de 1935. Decreto de 22 de Marzo de 1935. Aviso de 4 de Enero de 1936. Ordenanza de 24 de Febrero de 1936. Ordenanza de 29 de Abril de 1936. Ordenanza de 28 de Junio de 1936. Y Ordenanza de 18 de Agosto de 1936.

8 Por este Tratado se fijaron las definitivas fronteras que hoy tiene la República de Guinea Ecuatorial, por lo cual Espafia paś de tener un territorio superior a 200.000 kilómetros cuadrados, que inclulan parte de Gabón y Camerún a solo 20.000 . 
CONVENIO ENTRE LA FEDERACIÓN DE NIGERIA Y LA PROVINCIA ESPAÑOLA DEL GOLFO DE GUINEA

9 Liberia, fundada en 1822 por negros liberados de Estados Unidos y dependiente de este pals hasta su independencia en 1847.

10 Este Tratado fue firmado el 6 de septiembre de 1957 entre el Gobierno de la Federación de Nigeria, con la autoridad y consentimiento del Gobierno de Gran Bretaña e Irlanda del Norte y el Gobierno General de la Provincia Española del Golfo de Guinea, con la autoridad del Estado Espafiol.

11 Calabar es una región y una ciudad del Sureste de Nigeria que dista apenas unas millas de Fernando Poo.

12 Despacho número 169 de 12 de marzo de 1962 de la Embajada de España en Lagos. AMAE. Madrid 1962.

13 Abubakar fue el primer Primer Ministro de la Federación de Nigeria después de su independencia en octubre de 1960.

14 Despacho número 46 de 15 de septiembre de 1961 de la Embajada de Espana en Lagos. AMAE. Madrid, 1961.

15 En este Despacho de nuestro Embajador en Lagos se emplean términos y maneras que nos recuerdan muchoa los empleados por el Generalísimo.

16 Despacho número 169 de 12 de marzo de 1962 de la Embajada de Esparia en Lagos. AMAE. Madrid, 1962.

17 En el apéndice documental adjunto se puede encontrar el texto completo de dicho Tratado.

18 En todos los convenios suscritos con anterioridad el plazo era de dos afios prorrogables.

19 Despacho número 36 de 12 de agosto de 1961 de la Embajada de Espafía en Lagos. AMAE. Madrid, 1961.

20 Despacho número 247 de 7 de julio de 1962 de la Embajada de Espafia en Lagos. AMAE. Madrid, 1962.

21 Despacho número 327 de 19 de noviembre de 1962 de la Embajada de España en Lagos. AMAE. Madrid, 1962.

22 Despacho número 51 de 31 de marzo de 1963 de la Embajada de Esparia en Lagos. AMAE. Madrid, 1963.

\section{B I B L I O G RA F I A}

ÁLVAREZ GARCIA, H. R. (1948). Historia de la acción cultural en la Guinea espatiola. IDEA. Madrid. 558 págs.

ARNALTE, A. (1993). Una expedición de 3.000 morenos (un proyecto de colonización de Fernando Poo en 1870. Rev. AEA. Vol. VII. Núms. 12-13. Madrid. Págs. 89-105.

A.S.E.A. (1958). Convenio entre La Federación de Nigeria y la Provincia española del golfo de Guinea. Imprenta de los misioneros. Santa Isabel. 16 págs.

BONELLI Y RUBIO, J. M. (1949). Diferencia del concepto económico en la colonización de Fernando Poo y Guinea continental Rev. AEA. Núm. 7. Madrid.

FERNANDEZ CLEMENTE, E. (1979). Un lugar al sol. Rev. Historia 16. Número extra DX. Madrid. Págs. 96-104.

LLOMPART AULET, S. (1946). Legislación de trabajo en los Territorios españoles del Golfo de Guinea. IDEA. Madrid. 